\title{
MODELLING OF NATURAL CONVECTION FLOWS WITH LARGE TEMPERATURE DIFFERENCES: A BENCHMARK PROBLEM FOR LOW MACH NUMBER SOLVERS. PART 1. REFERENCE SOLUTIONS
}

\author{
Patrick Le Quéré ${ }^{1}$, Catherine Weisman ${ }^{1}$, Henri Paillère ${ }^{2}$, Jan Vierendeels ${ }^{3}$, \\ ERIK Dick ${ }^{3}$, Roland Becker ${ }^{4}$, Malte BraACK ${ }^{4}$ And James LoCKE ${ }^{5}$
}

\begin{abstract}
There are very few reference solutions in the literature on non-Boussinesq natural convection flows. We propose here a test case problem which extends the well-known De Vahl Davis differentially heated square cavity problem to the case of large temperature differences for which the Boussinesq approximation is no longer valid. The paper is split in two parts: in this first part, we propose as yet unpublished reference solutions for cases characterized by a non-dimensional temperature difference of $0.6, \mathrm{Ra}=10^{6}$ (constant property and variable property cases) and $\mathrm{Ra}=10^{7}$ (variable property case). These reference solutions were produced after a first international workshop organized by CEA and LIMSI in January 2000, in which the above authors volunteered to produce accurate numerical solutions from which the present reference solutions could be established.
\end{abstract}

Mathematics Subject Classification. 65M50, 76M10, 76M12, 76M20, 76M22, 76R10.

Numerical workshop, Low Mach Number Flows Conference, June 21-25, 2004, Porquerolles, France.

\section{INTRODUCTION}

Heat transfer by natural convection and conduction in enclosures occurs in numerous practical situations: solar collectors, cooling of nuclear reactors, heat management in electronic equipment, etc. In many of these situations, the temperature difference is small enough that the flow may be modelled assuming that the flow is incompressible with constant physical properties and buoyancy effects modelled as a linear function of the temperature, the so-called Boussinesq approximation. However, for large temperature differences, the flow becomes compressible with a strong coupling between the continuity, the momentum and the energy equations through the equation of state, and its properties (viscosity, heat conductivity) also vary with the temperature, making the Boussinesq flow approximation inappropriate and inaccurate. Most references in the literature deal with Boussinesq type natural convection flows. There is a clear need therefore to have reference data (i.e. to validate CFD codes) for natural convection flows with large temperature differences, where low Mach number

Keywords and phrases. Natural convection, non-Boussinesq, low Mach number.

1 LIMSI, BP 133, 91403 Orsay Cedex, France. plq@limsi.fr; weisman@limsi.fr

2 CEA Saclay, DEN/DM2S/SFME,91191 Gif-sur-Yvette Cedex, France. henri.paillere@cea.fr

3 Ghent University, B-9000 Gent, Belgium. Jan.Vierendeels@UGent.be; erik.dick@UGent.be

4 Heidelberg University, Germany. malte.braack@iwr.uni-heidelberg.de

${ }^{5}$ U. Warwick and British Energy Generation Ltd. 


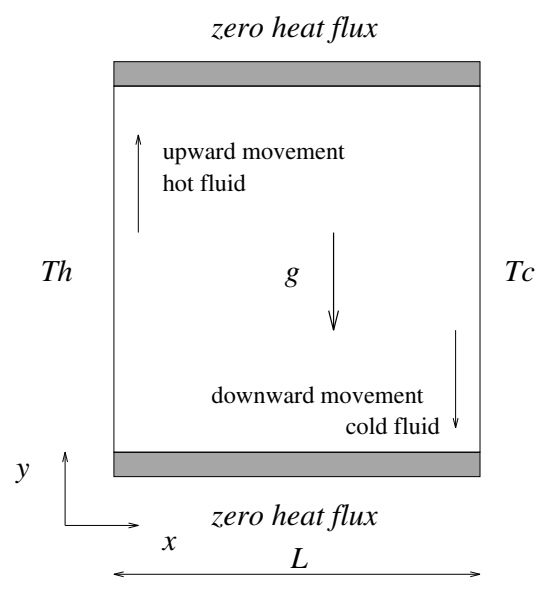

FiguRE 1. Differentially heated square cavity problem.

effects come into play. A test case was designed, extending the well-known de Vahl Davis benchmark problem [5] to cases with large temperature differences.

In January 2000, CEA and LIMSI organized a first international benchmark [11] dealing with this test case, with the objective of establishing reference (i.e. grid and model independent) solutions from a code-to-code comparison of various flow models and solvers. Twenty-two contributions were received and they enabled to point out several numerical difficulties - such as mass and energy conservation issues, post-processing of Nusselt numbers and CPU performance constraints. Following this benchmark, several participants performed further calculations to derive reference solutions. Their results which were not published, are presented here. In the second part of the paper, we will describe the results obtained in the framework of the conference on "Mathematical and numerical aspects of low Mach number flows" organized by INRIA and MAB in June 2004.

\section{A natural COnVection test-CASE}

\subsection{Description}

The test-case selected for the numerical conference organized by INRIA and MAB in June 2004 is the differentially heated square cavity problem depicted in Figure 1. It is similar to the well-known benchmark [5] for incompressible flow solvers which produced a set of reference solutions for different Rayleigh numbers Ra, ranging from $10^{3}$ to $10^{6}$ [4], later extended up to $10^{8}$ close to the end of the steady laminar regime [8]. We recall that for a perfect gas, the Rayleigh number is defined as

$$
\mathrm{Ra}=\operatorname{Pr} \frac{g \rho_{o}^{2}\left(T_{h}-T_{c}\right) L^{3}}{T_{o} \mu_{o}^{2}}
$$

where $\operatorname{Pr}$ is the Prandtl number ( 0.71 for air), $g$ is the gravity, $L$ the height of the cavity, $T_{h}$ and $T_{c}$ the hot and cold temperatures applied to the vertical walls, $T_{o}$ a reference temperature equal to $\left(T_{h}+T_{c}\right) / 2, \rho_{o}$ reference density corresponding to $T_{o}$, and $\mu$ the coefficient of viscosity at $T_{o}$. The temperature differences may be defined by the non-dimensional parameter $\epsilon$ :

$$
\epsilon=\left(T_{h}-T_{c}\right) /\left(T_{h}+T_{c}\right) .
$$

For small enough $\epsilon$, compressibility effects may be neglected, and incompressible flow models with the Boussinesq approximation are valid and accurate enough to compute the flow and the heat transfer to the walls [7]. 
The latter is characterized by the local and average Nusselt numbers $\mathrm{Nu}$ and $\overline{\mathrm{Nu}}$,

$$
\mathrm{Nu}(y)=\left.\frac{L}{k_{o}\left(T_{h}-T_{c}\right)} k \frac{\partial T}{\partial x}\right|_{w}, \quad \overline{\mathrm{Nu}}=\frac{1}{L} \int_{y=0}^{y=L} \mathrm{Nu}(y) \mathrm{d} y
$$

where $k(T)$ is the thermal conductivity and $k_{o}=k\left(T_{o}\right)$. For large temperature differences, the Boussinesq assumptions break down and one needs to resort to a compressible flow model, or since the Mach numbers remain small, to a low Mach number approximation model. Chenoweth and Paolucci [3] have studied in detail the effects of Ra and $\epsilon$ on flow patterns and heat transfer, but used temperature-dependent properties as well as varying Prandtl number. Polezhaev [14] studied the $\epsilon=0.2$ case with temperature-dependent properties and constant Prandtl number. Le Quéré et al. [9] have also studied the non-Boussinesq cases (up to $\epsilon=0.6$ ) with Sutherland's laws for viscosity and thermal conductivity.

\subsection{Governing equations}

We consider in this study the Navier-Stokes equations describing the flow of a compressible, calorically and thermally perfect gas, with constant Prandtl number. In conservative form, they read:

$$
\begin{aligned}
& \frac{\partial}{\partial t} \rho+\nabla \cdot(\rho \underline{u})=0 \\
& \frac{\partial}{\partial t}(\rho u)+\nabla \cdot(\rho \underline{u} \otimes \underline{u}+p \underline{\underline{I}})=\rho \underline{g}+\nabla \cdot \underline{\underline{\tau}} \\
& \frac{\partial}{\partial t}(\rho E)+\nabla \cdot(\rho \underline{u} H)=\nabla \cdot(k \nabla T)+\rho \underline{g} \cdot \underline{u} \\
& p=\rho R T=(\gamma-1) \rho\left(E-\frac{1}{2}\|\underline{u}\|^{2}\right)
\end{aligned}
$$

where $\rho, u, p, E$ and $H$ are respectively the density, velocity, pressure, specific total energy and specific total enthalpy. $\underline{g}$ and $\underline{\underline{\tau}}$ represent respectively the gravity and the viscous stress tensor,

$$
\underline{\underline{\tau}}=\mu\left(\nabla \underline{u}+\nabla^{T} \underline{u}-\frac{2}{3}(\nabla \cdot \underline{u}) \underline{I}\right) .
$$

$\mu$ and $k$ are respectively the dynamic viscosity and the heat conduction coefficients. At low Mach numbers, the dissipation term $\nabla \cdot(\underline{\underline{\tau}} \cdot \underline{u})$ in the energy equations may be neglected, and was omitted in the equation.

For non-conservative solvers based on low Mach number asymptotic approximations of the Navier-Stokes equations, the energy equation may be written in different forms, in terms of specific internal energy, enthalpy or temperature. For a perfect gas, and neglecting the energy dissipation term, the following form is advocated:

$$
\rho c_{p}\left(\frac{\partial}{\partial t} T+\underline{u} \cdot \nabla T\right)=\nabla \cdot(k \nabla T)+\frac{\mathrm{d} \bar{P}(t)}{\mathrm{d} t}
$$

where $\bar{P}(t)$ is the (spatially uniform) thermodynamic pressure in the domain, and $c_{p}$ is the specific heat at constant pressure, which may be expressed in terms of the ratio of specific heats $\gamma$ and the gas constant $R$, $c_{p}=\gamma R /(\gamma-1)$.

In the general case the evolution of $\bar{P}(t)$ is governed by a first order equation invoking the mass and heat fluxes at the boundary [12]. In the case of a cavity with impervious walls, this equation of evolution reduces to

$$
\frac{d \bar{P}}{d t}=(\gamma-1) \int_{\partial \Omega} \kappa \nabla T \cdot \mathbf{n} d \sigma
$$


Alternatively, $\bar{P}(t)$ can be obtained directly from the constraint of global conservation of mass contained in the cavity at initial time

$$
\bar{P}(t)=P_{0} \frac{\int \frac{1}{T_{0}} d v}{\int \frac{1}{T} d v}
$$

In this study, we shall assume either constant or temperature-dependent transport coefficients $\mu(T)$ and $k(T)$, given by Sutherland's law:

$$
\frac{\mu(T)}{\mu^{*}}=\left(\frac{T}{T^{*}}\right)^{\frac{3}{2}} \frac{T^{*}+S}{T+S}, \quad k(T)=\frac{\mu(T) \gamma R}{(\gamma-1) \operatorname{Pr}}
$$

with $T^{*}=273 \mathrm{~K}, S=110.5 \mathrm{~K}, \mu^{*}=1.68 \times 10^{-5} \mathrm{~kg} / \mathrm{m} / \mathrm{s}, \gamma=1.4$ and $R=287 \mathrm{~J} / \mathrm{kg} / \mathrm{K}$, and $\operatorname{Pr}=0.71$.

It is noted that at steady state, integration of the energy equation over the volume of the cavity represented in Figure 1 implies that the average Nusselt numbers on the vertical walls denoted by $L$ (left wall at $T_{h}$ ) and $R$ (right wall at $T_{c}$ ) are equal:

$$
\overline{\mathrm{Nu}}_{L}=\overline{\mathrm{Nu}}_{R} .
$$

\subsection{Initial conditions}

In each case, the problem is completely defined by the Rayleigh number, the value of $\varepsilon$ and the following coefficients:

$$
\begin{aligned}
P_{o} & =101325 \mathrm{~Pa} \\
T_{o} & =600 \mathrm{~K} \\
R & =287 \mathrm{~J} / \mathrm{kg} / \mathrm{K} \\
\rho_{o} & =\frac{P_{o}}{R T_{o}} \\
\operatorname{Pr} & =0.71 \\
\gamma & =1.4 \\
g & =9.81 \mathrm{~m} / \mathrm{s}^{2} .
\end{aligned}
$$

Spatially uniform initial conditions are imposed, $\forall(x, y) \in[0, L]^{2}$,

$$
\begin{aligned}
T(x, y) & =T_{o} \\
\rho(x, y) & =\rho_{o} \\
\bar{P}(t=0) & =P_{o} \\
u(x, y)=v(x, y) & =0 .
\end{aligned}
$$

\subsection{Boundary conditions}

On the hot wall, a temperature of $T_{h}=T_{o}(1+\varepsilon)$ is imposed, and on the cold wall, a temperature of $T_{c}=T_{o}(1-\varepsilon)$ is imposed. On the horizontal walls, adiabatic conditions are applied. On all walls, the no-slip condition is imposed for the velocity.

\subsection{Required results and workshop contributions}

Participants were asked to produce solutions for several test cases in a given format so as to be easily manipulated for the purpose of comparison. More than 25 contributions were received, which are reported in [11] and will not be discussed here. 


\section{Reference test CASEs AND CONTRIbutors}

During the round table discussion that took place in the January 2000 workshop, it was suggested that the comparison exercise would be incomplete if the opportunity was not taken to try to establish reference solutions for a few of the test cases. It was also proposed that these solutions should be established from a consensus between different contributors, rather than imposed from a single contributor, given the uncertainties in the underlying modelling approaches. Several contributors volunteered to perform additional calculations with the challenge to provide results of assessed quality that could be used to derive these reference solutions. These participants were asked to produce "grid-converged" results, or to ensure that their numerical results were sufficiently accurate by refining the mesh until the solutions varied no more (at least for the first four digits).

The three test cases which were selected are the following.

- Test case $\mathrm{T} 1: \mathrm{Ra}=10^{6}, \epsilon=0.6$ and constant properties $\mu=\mu^{*}$.

- Test case $\mathrm{T} 2: \mathrm{Ra}=10^{6}, \epsilon=0.6$ and Sutherland law $\mu=\mu(T)$.

- Test case T3: $\mathrm{Ra}=10^{7}, \epsilon=0.6$ and Sutherland law $\mu=\mu(T)$.

\subsection{Contributors}

\subsubsection{J. Locke, British Energy Generation Ltd and U. Warwick, UK}

The solutions were obtained by solving the steady-state equations, and using iteration to maintain the correct mass of fluid. The work was carried out using FEAT [6], a fluid modelling program developed by British Energy Generation Ltd. FEAT is a Finite Element code, which uses quadratic elements for accurate and smooth solutions. Upwinding is not usually needed, which allows $h^{4}$ truncation errors, and very fast mesh-convergence. The method of solution for a steady state of a non-linear equations system is based on Newton-Raphson iteration, and uses the frontal algorithm to solve the linearized system for each iteration. FEAT is used for low Mach number flows, but implements the full compressible form of the Navier-Stokes equations in order to deal with property dependence due to large temperature differences. The local pressure gradients in the equation for thermal energy conservation are ignored and, as well as this, the enthalpy is assumed to be a function of temperature alone. This means that the thermal energy is effectively decoupled from the energy in the flow field, and that FEAT cannot model sound waves.

\subsubsection{R. Becker and M. Braack, Heidelberg University, Germany}

The authors apply flow equations valid for low Mach number flow, and formulated in primitive variables where the total pressure is split in a hydrodynamical part and a thermodynamic part [1]. The hydrodynamical pressure is neglected in the equation of state, because it is several orders of magnitude smaller than the thermodynamic pressure, which is constant in space. The gas law becomes an algebraic equation for the density, in contrast to the full compressible formulation where it is the equation for the total pressure. The discretization is based on conforming bilinear Finite Elements on quadrilateral meshes. The Galerkin formulation is stabilized by introducing additional least-square terms. The discretized non-linear equations are solved by a quasi-Newton method and the resulting linear equations by multigrid iterations (V-cycle), with block-ILU smoothing. The results are obtained on equidistant tensor grids. Further details are available in [2].

\subsubsection{J. Vierendeels and E. Dick, Ghent University, Belgium}

An AUSM (Advection Upwind Splitting Method) based discretization method is implemented, with an explicit third-order upwind discretization for the convective part, a line-implicit central discretization for the acoustic part and for the normal diffusive fluxes. The tangential fluxes are discretized centrally and treated explicitly [15]. For vanishing Mach number, stabilization terms are added to the mass flux. A low Mach number preconditioning of the convective fluxes is also used. A semi-implicit line method in multistage form is used because of the explicit third-order discretization of the convective part. The multistage semi-explicit method is accelerated with the multigrid method, using a full approximation scheme in a W-cycle with six levels of grids. The pressure in each node is re-scaled after each MG cycle so as to enforce the mass conservation. 


\subsubsection{Weisman and P. Le Quéré, LIMSI, France}

The algorithm integrates in time the low Mach number equations formulated in primitive variables where the total pressure is split in a hydrodynamical part and a thermodynamic part. Time advancement is performed using a fractional time step method derived from the projection method used to compute incompressible flows. Spatial discretization uses a second order spatial approximation for both convective and diffusive fluxes on a standard finite volume staggered mesh. The momentum equations are advanced in time using an incremental ADI solver, using the old pressure field. A projection step is used to compute the velocity field with given divergence. This step involves the solution of a non separable Poisson equation, which is done using a multigrid algorithm. Spatial care was taken to ensure that all compatibility conditions were satisfied at the discrete level. Steady solutions were obtained by integrating long enough, and it was checked that the steady solutions were independent of the time step, thanks to the use of the incremental ADI solvers. Most computations were performed with Chebyshev and uniform grids in the horizontal and vertical direction, respectively.

\subsubsection{P. Le Quéré, LIMSI, France}

The test cases were computed using the algorithm described in [9]. This algorithm integrates the Low Mach number equations in unsteady form. The spatial approximation uses Chebyshev pseudo-spectral approximations in both spatial directions. The time stepping is second order and combines an explicit treatment of the convective terms with and implicit treatment of the diffusive terms. The resulting non separable Helmholtz equations are solved iteratively within each time step using an approximate factorization of the finite difference approximation of the Helmholtz operator as preconditioning. The divergence constraint is imposed using an Uzawa type iterative algorithm. Steady state solutions are obtained by integrating long enough.

\subsection{Accuracy assessment}

Assessing the quality and quantifying the accuracy of numerical solutions is a matter of utmost importance which is drawing increasing attention under the name of verification and validation. For most general programs and configurations this is a very complex process which is described for instance in [10]. The problem at hand belongs to a class of configurations for which the process of accuracy assessment involves three different aspects:

- making sure that the computer program is bug free;

- making sure that the steady state solution has been obtained;

- quantifying the spatial discretization error.

Aside from obvious coding mistakes which should never be overlooked, the first point is related to the consistency of the overall discrete algorithm with the continuous equations, and will not be discussed here.

The second point is particularly important for unsteady algorithms where steady states are obtained by letting time run to infinity. Infinity corresponds to the time scale for the flow establishment in the cavity and it is known [13] that this time scale is a diffusive time scale corresponding to the time needed to damp the internal waves in the cavity core which scales like $\mathrm{Ra}^{1 / 2}$ in the dimensionless units considered here. For explicit or semi-implicit schemes with the usual stability strains, integrating for such a long time can require hundreds of thousands of time steps, resulting in very long CPU times.

Quantifying the spatial discretization error in order to produce a solution of increased accuracy can usually be done through a Richardson extrapolation. Richardson extrapolation consists of computing solutions on different meshes, determining the leading order of the truncation error and extrapolating these solutions to zero mesh size. Let $f_{h}, f_{h^{\prime}}, f_{h^{\prime \prime}}$ be the numerical approximations for a given scalar quantity $f$ computed on three corresponding grids of of mesh size $h \leq h^{\prime} \leq h^{\prime \prime}$ respectively with $\frac{h}{h^{\prime}}=\frac{h^{\prime}}{h^{\prime \prime}}$. Assuming that these grid meshes fall within the asymptotic convergence region with the truncation error at leading order characterized by $h^{\alpha}$, it follows that:

$$
\begin{aligned}
f_{h} & =f+C h^{\alpha} \\
f_{h^{\prime}} & =f+C h^{\prime \alpha} \\
f_{h^{\prime \prime}} & =f+C h^{\prime \prime \alpha} .
\end{aligned}
$$


TABLE 1. Reference results obtained in 2000.

\begin{tabular}{|c|c|c|c|c|c|c|}
\hline & $\begin{array}{l}\text { Locke } \\
(\mathrm{FE})\end{array}$ & $\begin{array}{c}\text { Becker } \\
\& \text { Braack }(\mathrm{FE})\end{array}$ & $\begin{array}{l}\text { Vierendeels } \\
\& \text { Dick (FV) }\end{array}$ & $\begin{array}{c}\text { Weisman } \\
\text { \& Le Quéré }(\mathrm{FV})\end{array}$ & $\begin{array}{l}\text { Le Quéré } \\
\text { (Spectral) }\end{array}$ & $\begin{array}{c}\text { Reference } \\
\text { Solution }\end{array}$ \\
\hline \multicolumn{7}{|c|}{ Test case $\mathrm{T} 1$ ( $\mathrm{Ra}=10^{6}, \epsilon=0.6$ and constant properties $)$} \\
\hline $\mathrm{Nu}(\mathrm{h})$ & 8.859785 & 8.859780 & 8.859783 & 8.859781 & 8.859777 & 8.85978 \\
\hline $\mathrm{Nu}(\mathrm{c})$ & 8.859789 & 8.859780 & 8.859783 & 8.859780 & 8.859777 & 8.85978 \\
\hline $\mathrm{P} / \mathrm{Po}$ & 0.8563379 & 0.8563379 & 0.8563383 & 0.8563376 & 0.8563380 & 0.856338 \\
\hline Mesh & $160 \times 160$ & $4 \times 10^{6}$ d.o.f. & $512 \times 512$ & $512 \times 512$ & $80 \times 80$ & \\
\hline \multicolumn{7}{|c|}{ Test case $\mathrm{T} 2\left(\mathrm{Ra}=10^{6}, \epsilon=0.6\right.$ and Sutherland law $)$} \\
\hline $\mathrm{Nu}(\mathrm{h})$ & 8.686678 & 8.686605 & 8.686587 & 8.686583 & 8.686581 & 8.6866 \\
\hline $\mathrm{Nu}(\mathrm{c})$ & 8.686617 & 8.686605 & 8.686611 & 8.686583 & 8.686581 & 8.6866 \\
\hline $\mathrm{P} / \mathrm{Po}$ & 0.9244872 & 0.9244874 & 0.9244877 & 0.9244872 & 0.9244873 & 0.924487 \\
\hline Mesh & $160 \times 160$ & $4 \times 10^{6}$ d.o.f. & $512 \times 512$ & $1024 \times 1024$ & $80 \times 80$ & \\
\hline \multicolumn{7}{|c|}{ Test case $\mathrm{T} 3\left(\mathrm{Ra}=10^{7}, \epsilon=0.6\right.$ and Sutherland law $)$} \\
\hline $\mathrm{Nu}(\mathrm{h})$ & 16.240740 & 16.241060 & 16.2409905 & 16.24097 & & 16.2410 \\
\hline $\mathrm{Nu}(\mathrm{c})$ & 16.240740 & 16.241060 & 16.2411507 & 16.24097 & & 16.2410 \\
\hline $\mathrm{P} / \mathrm{Po}$ & 0.9226343 & 0.9226358 & 0.92263384 & 0.92263384 & & 0.92263 \\
\hline Mesh & $160 \times 160$ & $4 \times 10^{6}$ d.o.f. & $512 \times 512$ & $1024 \times 1024$ & & \\
\hline
\end{tabular}

Combining these equation leads to the values of $\alpha$ and $C$ :

$$
\alpha=\frac{\ln \left(\frac{f_{h}-f_{h}^{\prime}}{f_{h}^{\prime}-f_{h}^{\prime \prime}}\right)}{\ln \left(\frac{h}{h^{\prime}}\right)}, \quad C=\frac{f_{h}-f_{h}^{\prime}}{h^{\alpha}\left(1-\left(\frac{h^{\prime}}{h}\right)^{\alpha}\right)} ;
$$

which allows for the computation of the extrapolated value for $f$. This procedure works if higher order terms are really negligible i.e. if $f_{h}=f+C h^{\alpha}$ is a good approximation of the truncation error for the coarser grid (see [10] for a thorough discussion).

\section{Reference solutions (2000)}

Table 1 summarizes the converged results obtained for each of the three test cases from the 5 different contributions. As can be seen, an excellent agreement is obtained between the different contributions and the last column provides a reference solution with all digits exact given the usual rounding conventions. The accuracy of reference solution $\mathrm{T} 1$ is close to $10^{-6}$, whereas that of solutions $\mathrm{T} 2$ and $\mathrm{T} 3$ is slightly lower and around $10^{-5}$. It is to be noted that establishing these reference solutions has required computing solutions over very fine meshes in most cases (except for Locke) and that significant computing resources were involved. It should also be noted that the level of agreement is quite exceptional given the fact that all these contributions do not solve exactly the same physical model (some use the full compressible equations while others use the low-Mach number equations). The last comment is that one should not try to derive reference solutions of 
infinite accuracy, because the differences in the models, fully compressible or low-Mach number, will eventually show up in the solutions, at accuracy levels of $M a^{2}$, that is around $10^{-7}$ or $10^{-8}$. This still leaves some room for improvement for cases T2 and T3.

\section{Conclusion}

Reference solutions have been derived for three test cases of steady natural convection flows of gases under large temperature differences. These reference solutions can be used in the process of validation and verification of CFD codes at the elementary level, before addressing more complex physical configurations.

\section{REFERENCES}

[1] R. Becker, M. Braack and R. Rannacher, Numerical simulation of laminar flames at low Mach number with adaptive finite elements. Combustion Theory and Modelling, Bristol 3 (1999) 503-534.

[2] R. Becker, M. Braack, Solution of a stationary benchmark problem for natural convection with high temperature difference. Int. J. Thermal Sci. 41 (2002) 428-439.

[3] D.R. Chenoweth and S. Paolucci, Natural Convection in an enclosed vertical air layer with large horizontal temperature differences. J. Fluid Mech. 169 (1986) 173-210.

[4] G. de Vahl Davis, Natural convection of air in a square cavity: a benchmark solution. Int. J. Numer. Methods Fluids 3 (1983) 249-264.

[5] G. de Vahl Davis and I.P. Jones, Natural convection of air in a square cavity: a comparison exercice. Int. J. Numer. Methods Fluids 3 (1983) 227-248.

[6] FEAT User Guide, Finite Element Analysis Toolbox, British Energy, Gloucester, UK (1997).

[7] D.D. Gray and A. Giorgini, The Validity of the Boussinesq approximation for liquids and gases. Int. J. Heat Mass Transfer 15 (1976) 545-551.

[8] P. Le Quéré, Accurate solutions to the square differentially heated cavity at high Rayleigh number. Comput. Fluids 20 (1991) $19-41$.

[9] P. Le Quéré, R. Masson and P. Perrot, A Chebyshev collocation algorithm for 2D Non-Boussinesq convection. J. Comput. Phys. 103 (1992) 320-335.

[10] W.L. Oberkampf and T. Trucano, Verification and validation in Computational Fluid Dynamics. Sandia National Laboratories report SAND2002-0529 (2002).

[11] H. Paillère and P. Le Quéré, Modelling and simulation of natural convection flows with large temperature differences: a benchmark problem for low Mach number solvers, 12th Séminaire de Mécanique des Fluides Numérique, CEA Saclay, France, 25-26 Jan., 2000.

[12] S. Paolucci, On the filtering of sound from the Navier-Stokes equations. Sandia National Laboratories report SAND82-8257 (1982).

[13] J.C. Patterson and J. Imberger, Unsteady natural convection in a rectangular cavity. J. Fluid Mech. 100 (1980) 65-86.

[14] V.L. Polezhaev, Numerical solution of the system of two-dimensional unsteady Navier-Stokes equations for a compressible gas in a closed region. Fluid Dyn. 2 (1967) 70-74.

[15] J. Vierendeels, K. Riemslagh and E. Dick, A Multigrid semi-implicit line-method for viscous incompressible and low-Mach number flows on high aspect ratio grids. J. Comput. Phys. 154 (1999) 310-341. 